\title{
AOR
}

Selected Papers of \#AolR2020:

The $21^{\text {st }}$ Annual Conference of the

Association of Internet Researchers

Virtual Event / 27-31 October 2020

\section{MAPPING VALUE(S) IN AI: THE CASE OF YOUTUBE}

\author{
Bernhard Rieder \\ University of Amsterdam \\ Geoff Gordon \\ University of Amsterdam \\ Giovanni Sileno \\ University of Amsterdam
}

\begin{abstract}
This paper presents a multidisciplinary approach (media studies, computer science, and legal scholarship) for the analysis of systems that rely on Al components as central components of their design. Taking recommender systems more generally and the one built by YouTube more specifically, we develop a methodology for conceptualizing and studying the broad array of "ideas", "norms", or "values" such systems mobilize. Instead of limiting ourselves to a narrow understanding of these terms, we take into account, for example, translations from economic models, social theories, legal requirements, ethical principles, technical knowledge, experiential evaluations, or other constructs used to define and justify design goals and decisions that shape the production of technical objects and, consequently, the object themselves. In this paper we discuss three directions for analysis and present first results. Investigating technical knowledge includes the study of scholarly literature and experimentation with concrete objects such as LensKit for Python to understand the "ambient" knowledge and normativity engineers and designers draw on. Investigating local circumstances involves ethnographic analysis, but also the reconstruction of the business models and legal environment that weigh on YouTube's design. Analyzing a system in use can draw on technical observation, via scraping or API data, of the actual dynamics of recommendation that emerge when users enter into the equation. Taken together, these three approaches can "encircle" the various moments where value(s) are shaped and put into work in the context of systems where direct access to specifications is improbable.
\end{abstract}

Suggested Citation (APA): Rieder, B., Gordon, G., Sileno, G. (2020, October) Mapping Value(s) in Al: The Case of Youtube. Paper presented at AolR 2020: The $21^{\text {th }}$ Annual Conference of the Association of Internet Researchers. Virtual Event: AolR. Retrieved from http://spir.aoir.org. 


\section{Introduction}

Over the recent decade, systems integrating Al components have both proliferated on the Internet and come under increased scrutiny. Particularly in the context of large online platforms, there is a stark contrast between anxieties about negative effects and structural opacity, that is, the impossibility to get a firm grip on the mechanisms at work. While work on "algorithmic accountability" (e.g. Diakopoulos, 2015) has made great strides and there is a long tradition on "values in design" (cf. Knobel \& Bowker, 2011) to build on, the question of normativity in complex computing systems operating within specific social contexts remains challenging.

This paper uses the case of recommender systems more generally and the one built by YouTube more specifically to develop a methodology for conceptualizing and studying the broad array of "ideas", "norms", or "values" such systems mobilize. But what do these terms mean in the context of technical systems? Are they simple transpositions of non-technical constructs into technical form? Instead of limiting ourselves to a narrow understanding, we situate algorithmic systems within a complex networks of "value(s)" understood, for example, as translations from economic models, social theories, legal requirements, ethical principles, technical knowledge, experiential evaluations, or other constructs used to define and justify design goals and decisions - that shape the production of technical objects and, consequently, the object themselves. Our multidisciplinary methodology draws on media studies, computer science, and legal scholarship and follows three pathways, analyzing technical knowledge, local circumstances, and systems in use. This paper sketches each direction and presents a number of early results from the larger project.

\section{Investigating Technical Knowledge}

This part of our methodology concerns the investigation of recommender systems as a field of knowledge as well as an archive of existing "algorithmic techniques". It draws on the extensive (academic) literature available, but also engages technicity more directly through investigation and experimentation with existing code.

Historical investigation uncovers precursors (e.g. the work of Elaine Rich in the early 1980s), early examples of contemporary work (e.g. GroupLens), and the construction of different technical trajectories (e.g. content-based vs. collaborative filtering).

Discourse analysis draws on a corpus of "standard" literature ( to reconstruct the conceptual apparatus, methodological horizon, and state of the art in the field of recommender systems. For example, the Handbook by Ricci et al. (2015) specifies 14 criteria or properties that could be used to decide whether a recommendation system is performing according to its goals: user preference, prediction accuracy, coverage, confidence, trust, novelty, serendipity, diversity, utility, risk, robustness, privacy, adaptivity, and scalability. Each one of these dimensions involves normative reasoning, including justifications, but also concrete ways to evaluate each of them in practice.

Experimental analysis proceeds in line with recent efforts in the humanities to establish how complex algorithms "think" (cf. Burrell, 2016) and uses existing code modules (e.g 
LensKit for Python) to experiment with both code inspection and behavioral tweaking. The goal is to approach a concrete technical object to build an intuition for its epistemological character and possibilities for variation.

\section{Investigating Local Circumstances}

While technical knowledge, practices, and norms create an environment for concrete projects to draw on, these systems involve design decisions that rely heavily on local circumstances. Broad understanding of recommender systems and the fields they connect with can help frame investigations of actual systems, but another set of approaches is required to capture a concrete system, like YouTube's. Here, we take inspiration from the technique of "encircling", recently developed in the context of security studies, where secrecy and obfuscation are a constant reality that researchers have to deal with through "a lateral, multipronged, creative, iterative approaching of secret sites, confidential materials and classified practices" (De Goede et al., 2019, p. 14)

Ethnographic investigation indeed has to deal with the fact that participant observation and even interviews are hard to realize for YouTube's recommendation system. But practitioners often continue to speak publicly and in the case of YouTube, we can rely on academic publications (e.g. Covington et al., 2016) as well as so-called "grey literature", such as documentation, media interviews, or business reports, as a means to learn about the system under scrutiny and the way its creators present and justify it.

Legal analysis reconstructs the legal and regulatory environment YouTube operates in. This includes the broader provisions concerning online platforms, but also more specific legislation, such as the US Children's Online Privacy Protection Act, which imposes a number of constraints on the company.

Analysis of business practices constitutes a second approach to understanding a concrete recommender system and this involves an analysis of the organization that designs and maintains the system, in particular its business model and the incentive structures that model generates. We certainly accept that the 'bottom line' is not the only motivation for a firm and there are many ways to drive and develop income structures. But it should be clear that organizations are goal-driven, and these goals will exercise a constant influence on all design decisions.

\section{Investigating a System in Use}

The final part of our methodology builds on the recognition that platforms hosting billions of users are not in full control over what happens within their systems. After all, YouTube's recommender system fundamentally draws on actual practices of uploading videos, watching, liking, commenting, and so forth. This means that what is actually recommended is a distributed accomplishment rather than a mere effect of a technical system.

Technical observation is a means to study a system in use empirically. The approach starts from the idea that systems that are publicly accessible on the internet can be 
"observed" in the sense that both inputs and outputs can be collected at a variable degree. This does not give us 'hard' insights into the technical system but allows us to make certain claims about its behavior: What is actually being recommended? How do recommendations evolve over time? Can we observe a trend toward popular content or is the system driving toward niche content?

\section{Conclusion}

While this paper focuses on recommender systems and takes YouTube as its main example, its purpose is to prototype and present a multidisciplinary methodology for large-scale platforms that incorporate Al components as central tools of governance. It starts from the idea that technical knowledge forms the basis of technical design and seeks to bring these "ambient" norms into conversation with the local circumstances of a concrete system that includes a company, different groups of users, and the dynamics that play out over time.

\section{References}

Burrell, J. (2016). How the machine 'thinks': Understanding opacity in machine learning algorithms. Big Data \& Society, 3(1).

Covington, P., Adams, J., \& Sargin, E. (2016). Deep Neural Networks for YouTube Recommendations. Proceedings of the 10th ACM Conference on Recommender Systems - RecSys '16, 191-198.

De Goede, M., Bosma, E., \& Pallister-Wilkins, P. (Eds.). (2019). Secrecy and Methods in Security Research: A Guide to Qualitative Fieldwork. Routledge.

Diakopoulos, N. (2015). Algorithmic Accountability: Journalistic investigation of computational power structures. Digital Journalism, 3(3), 398-415.

Knobel, C., \& Bowker, G. C. (2011). Values in design. Communications of the ACM, 54(7), 26-28.

Ricci, F., Rokach, L., \& Shapira, B. (Eds.). (2015). Recommender systems handbook (2nd Edition). Springer. 